CHAPTER XI

QUASI-STELLAR OBJBCTS: DISTRIBUTION AND GBNERAL PROPERTIES 


\title{
COSMIC DISTRIBUTION OF OPTICALLY SELECTED QUASARS
}

\author{
Maarten Schmidt \\ Palomar Observatory \\ California Institute of Technology \\ Pasadena, CA 91125, USA
}

\begin{abstract}
Counts of optically selected quasars as a function of magnitude and redshift show the effects of strong evolution. If quasars have relatively short life times, then the observed numbers at a given redshift are mostly determined by their birth rate and mean luminosity over their lifetime. In this case the evolution of the luminosity function can be described by density evolution, where the rate of evolution may depend on luminosity and other properties. On the other hand, if all quasars were formed at large redshift and have been decaying in luminosity since that time, then the evolution of the luminosity function is best described in terms of luminosity evolution. We discuss some of the consequences of luminosity evolution for the mass of quasars and for the X-ray background.

We explore the observational aspects of the redshift cutoff of quasars. The situation is complicated by the unavoidable bias in slitless surveys against weak-line objects. Since quasar emission lines show a wide range of equivalent widths, a spectral survey will be characterized by a distribution of limiting continuum magnitudes rather than by a single value. The decline in the space density of quasars at large redshift may depend on luminosity, and may also have structure, such as a steep drop, but not a total cutoff, in density at a redshift near 3 .
\end{abstract}

\section{INTRODUCTION}

The cosmic distribution of quasars shows evidence for strong evoluticn of quasar properties with redshift. This finding is based on the statistics of quasars in both apparent magnitude and redshift. The evolution exhibits itself in a redshift dependence of the luminosity function, which mediates all quasar statistics.

We will discuss the observational evidence for evolution and in particular the concepts of density and luminosity evolution. We also review surveys of high redshift quasars in connection with the decline of quasar numbers around a redshift of 3 . 


\section{QUASARS SELECTED BY COLOR}

For redshifts below 2.2, the ultraviolet excess is an effective way to select quasar candidates. Spectroscopic observations are required to confirm the quasar identification and to obtain the redshift. For a recent review, the reader is referred to Green (1986), who collected all available samples based on spectroscopically confirmed redshifts and showed the luminosity function in seven intervals of redshift. Since that time, Koo et al. have published a survey for quasars to $B=22.5$ selected on the basis of multiple colors, variability and proper motions. Boyle et al. report in this volume on a survey of about 170 quasars with $\bar{B}<20.9, z<2.2$, which will add much weight to the derivation of the luminosity function.

The observed variation of the luminosity function with redshift may be alternatively described as an increase of density with redshift at given luminosity, or as an increase in luminosity with redshift.

At this stage, it is useful to remember that the observed luminosity function represents the properties of the ensemble of quasars. The number and luminosities of quasars at any given time are determined by the birth rate of quasars and their subsequent light curve $L(t)$. Disentangling the contributions of birth rate and light curve will in general be difficult. If all quasars had individual light curves showing decay at a 30 million year time scale, we would not be able to derive that from a luminosity function that shows variation on a time scale of around a billion years.

\subsection{Density Evolution}

If we assume that quasars have life times or time scales much shorter than the age of the universe, then their observed numbers mostly reflect their birth rates integrated over their life time. If the birth rate varies with cosmic time, density evolution will result.

The first evidence for evolution of optically selected quasars (Schmidt 1970) was based on only 23 objects. With so few objects in hand, little else could be done than to parametrize the co-moving space density as a function of redshift only. This scenario is now called pure density evolution (PDE).

In the early 1980s, sufficient survey material became available that the density increase could be investigted in more detail. Assuming that the co-moving density changed with cosmic time as exp(-kt), Schmidt and Green (1983) discussed the available samples. They found that $k$ could be well determined for quasars of $M(B)$ around -27. Applying this same value of $k$ to quasars regardless of their luminosity yielded an overestimate of quasar numbers with $B<21$ by a factor of 20 . Since most of these quasars are less luminous than $M(B)=-27$, $k$ had to be smaller for quasars of lower luminosity. This started the concept of luminosity-dependent density evolution (LDDE). Schmidt and Green (1983) opted for a simple linear dependence of $k$ on $M(B)$, since the available material did not allow more detailed conclusions. On the basis of new material, including that presented by Boyle et al, in this volume, the dependence of $k$ on $M(B)$ may have to be reevaluated. 
A11 recent authors in the field agree the PDE is inconsistent with the observations. Some authors have argued that LDDE does not fit the observations. If so, that may be a consequence of new observational material that was not available at the time of the Schmidt and Green (1983) paper. In principle, LDDE can fit any observations, since it simply describes the density increase at given luminosity--if there is a need, the density law can be made to depend on other properties beyond luminosity.

\subsection{Luminosity Evolution}

The alternative approach to the one sketched above is to assume quasars are long-lived and to interpret the change in the luminosity function as a consequence of evolution of luminosity. This follows if one assumes that all quasars were formed at an early epoch. In that case, the total quasar density does not change and the luminosity function will shift in the luminosity coordinate $\mathrm{L}$, reflecting the light curve $\mathrm{L}(\mathrm{t})$ of individual quasars. This is the case of pure luminosity evolution (PLE), introduced by Mathez (1978).

Boyle et al. (this volume) find that for redshifts below 2.2, PLE provides a good representation of the available statistics. The luminosity evolution required between $z=0$ and $z=2.2$ amounts to about 5 magnitudes. In other words, some 5 magnitudes of the bright end of the local luminosity function of Seyfert galaxies is shifted into the quasar luminosity range at redshift 2.2 .

There are at least two problems with the concept of long-lived quasars and PLE that have to be addressed. The first one concerns the X-ray background. As shown by Schmidt and Green (1986), even if there is no evolution at all, Seyfert galaxies with luminosities $M(B)>-23$ contribute $29 \%$ of the $X$-ray background. If there is evolution of Seyfert galaxies as required in PLE, then the X-ray background contributed by Seyferts far exceeds the total measured background.

The second concern has to do with the total mass of quasars. In the case of PLE, a quasar like 3C 273 will have radiated around $3 \times 10^{65}$ ergs over the past 10 billion years. The corresponding mass based on a $10 \%$ efficiency of accreted mass into radiated energy is around $10^{12}$ solar masses. The corresponding Schwarzschild radius of about a light month may be in conflict with the time scale of observed quasar variability.

Problems with either the X-ray background or the mass of quasars can be avoided if the life time of quasars is much less than that of the universe. To maintain PLE, dying quasars would have to be replaced in equal numbers by newly born ones to keep the number constant. Such an accidental situation is avoided in LDDE, where there is no imposed equality between the number of births and deaths.

\section{QUASARS SELECTED BY EMISSION LINES}

For redshifts larger than 2.2, Lyman-alpha emission enters the B filter and the ultraviolet excess usually disappears. Most surveys of quasars of larger redshift are based on slitless spectral surveys, in which 
selection of candidates is made on the basis of the presence of 1 ine emission.

Such surveys have yielded large numbers of quasars. The most remarkable result that has emerged is that quasars of very high redshift are rare and that there may be a redshift cutoff. The first convincing evidence for a decrease in quasar density (with increasing redshift) came from Osmer (1982) who failed to find any quasars with redshift in the range 3.7-4.7 in an area of 5 square degrees down to magnitude 20 . No quasars with redshifts substantially larger than 4 have been found yet. Since the quasars with the largest observed redshifts have magnitudes as bright as 17 or 18, it is clear that such quasars could be easily detected at larger redshifts if they existed.

Veron (1986) has reviewed results of published emission-line surveys and concludes that the quasar density rises with redshift up to 2.45 , drops by a factor of 5 , then rises with redshift up to 3.8 to drop steeply once more. On the other hand, Hazard and McMahon (1985) state, on the basis of a UK Schmidt telescope objective prism plate, that the density of quasars peak at a redshift of 2 .

The reliability of emission-lines surveys has sometimes been questioned. In particular, there is uncertainty about their completeness or their limiting magnitude (Clowes 1981; Smith 1983; Veron 1983). This is best illustrated by a comparison of the surface densities of quasars found in Schmidt surveys and in 4-meter telescope surveys, which differ by a factor of up to 10 at overlapping magnitude (Osmer 1980). As pointed out by Clowes (1981) and Smith (1983), faint objects in a survey will only be picked up if the emission line is strong.

Schmidt, Schneider, and Gunn (1986a,$\underline{b})$ use a strict algorithm for the acceptance of an emission-line object as a candidate quasar, namely that the emission line be detected with a given minimum signal-to-noise ratio and that it have a minimum observed equivalent width. Since a given emission line exhibits a large range of equivalent widths in quasar spectra, a survey at any given redshift has a distribution of limiting magnitudes, corresponding to the equivalent width distribution. In addition, due to the response function of the detector, the limiting magnitude at given equivalent width is a function of wavelength and hence of redshift.

The complexities of a survey that has a distribution of limiting magnitudes, rather than a single value, makes it somewhat difficult to construct luminosity functions from the survey observations in a straightforward manner. It is, of course, possible to compare observed numbers of quasars at different redshifts with predictions based both on luminosity function models and on an adopted distribution of the (zero redshift) equivalent widths of the main emission lines.

Schmidt et al, (1986a,$\underline{b})$ used such a procedure to compare results from two surveys for high redshift quasars with a smooth extrapolation of luminosity function models constructed for $z<2.2$, discussed in the preceding section. No quasars with $z>3.5$ were found in these two surveys, whereas the extrapolated models predicted that around 70 should have been observed. This is entirely consistent with Osmer's (1982) finding. Schmidt et al. also did not find any quasars with redshift over 3 , where the extrapolated models would predict that 20 should have 
been observed. This was rather surprising, since general quasar catalogues contain some 50 quasars with redshifts larger than 3 , based on slit spectroscopy.

There is no evidence at present that an abrupt shift in the distribution of equivalent widths of emission lines causes the shortfall of redshifts larger than 3 in systematic surveys. Since almost a11 high-redshift quasars in the quasar catalogues are quite bright, we initially proposed (Schmidt et al. 1986a) that the redshift at which quasar densities reach a maximum and turn around might be a function of luminosity, in the sense that quasars of higher luminosity have a larger redshift cutoff. When a survey that went less deep over a larger area still produced no quasars with redshift over 3 (Schmidt et al. 1986 b), we suggested the possibility that the density of quasars might exhibit a steep drop, by a factor of 10 or 20 , at a redshift near 3 , with a subsequent slow decline to zero at a redshift beyond 4 . This could explain why small samples yield no quasars with $z>3$, while a collection of several thousand objects such as the entire quasar catalogue yields a substantial number.

Much additional work will be needed to clarify the situation. It is essential that further spectroscopic surveys of quasars address the detectability of the emission lines in quantitative fashion. Improved understanding of the evolution and the redshift cutoff of quasars will depend critically on insight into the completeness of quasar surveys.

This research was supported in part by the National Science Foundation under grant AST-8314134.

Note added October 15, 1986 :

Schmidt, Schneider and Gunn have recently observed slit spectra of emission-1ine candidates of a 4-Shooter spectroscopic transit survey covering 14 square degrees. A first list of 75 candidates was selected from a total of 120,000 spectra observed. A total of 16 quasars was found, of which 9 have redshifts between 3.0 and 3.8 . Schmidt et al. conclude that there is no evidence for a sharp drop in quasar density at a redshift just below 3: instead, the observations suggest that there is a gradual decline of quasar density beyond a redshift of 3 .

\section{REFERENCES}

Clowes, R.G. 1981, M.N.R.A.S., 197, 731.

Green, R.F. 1986, in IAU Symposium 119 on Quasars, held in Bangalore, December 1985.

Hazard, C., and McMahon; R. 1985, Nature, 314, 238.

Mathez, G. 1978, Astron. Atrophys., 68, 17.

Osmer, P.S. 1980, Astrophys. J. Supp1., 42, 523.

Osmer, P.S. 1982, Astrophys. J., 253, 28.

Schmidt, M. 1970, Astrophys. J., 162, 371.

Schmidt, M., and Green, R.F. 1983, Astrophys. J., 269, 352.

Schmidt, M., and Green, R.F. 1986, Astrophys. J., 305, 68.

Schmidt, M., Schneider. D.P., and Gunn, J.E. 1986a, Astrophys J., $306,411$. 

Schmidt, M., Schneider, D.P., and Gunn, J.E. 1986므, Astrophys. J.,

Smith, M.G. 1983, in 'Quasars and Gravitational Lenses', Proc. of the 24th Liège Colloquium (Université de Liège), p.4.

Veron, P. 1983, in 'Quasars and Gravitational Lenses', Proc. of the 24th Liège Colloquium (Université de Liège), p. 210.

Veron, P. 1986, Astron. Astrophys. (submitted).

\section{DISCUSSION}

BOLDT: What is the value of $\mathrm{q}_{\mathrm{o}}$ that you assumed in evaluating the number of quasars expected at high redshifts?

SCHMID?: I assumed $\mathrm{q}_{\mathrm{O}}=0.5$; but the predictions differ little for $\mathrm{q}_{\mathrm{o}}=0.1$.

SEITI: Referring to your statement that Sy 1 should not evolve otherwise their integrated emission would exceed the XPB, it should be remembered that in the case of luminosity evolution models, this may not be the case because it appears that the X-ray 11minosity does not increase as fast as the optical luminosity $\left(\mathrm{L}_{\mathrm{x}} \propto \mathrm{L}_{0} 0.7-0.8\right)$.

SCHMIDT: Since there is a large observed scatter of the $\left(\mathrm{L}_{\mathrm{x}}, \mathrm{L}_{0}\right)$ correlation, luminosity evolution may not follow the mean rêlation. Even if the X-ray luminosity evolution is so reduced, the XRB predicted for Seyferts on the basis of optical PLE still exceeds the observed background.

LONCAIR: I believe important information on the cut-off at large redshifts is provided by radio source surveys which are not subject to the awkward selection effects which beset the emission line surveys. Peacock, Dunlop and their colleagues have been carrying out a survey of about 180 radio sources selected at $2.7 \mathrm{GHz}$ with flux densities $\mathrm{S}_{2,7} \geq 0.1 \mathrm{Jy}$. This sample is designed to be most sensitive to the presence or absence of radio sources with flat radio spectra with $z \geq 4$. The identifications and spectra of the sources in this sample are well advanced and already it is clear that there are few objects with very large redshifts in the sample. A banded $V / V_{\text {max }}$ test shows that at redshifts greater than about 2, the comoving space density of radio quasars decreases from a redshift of 2 to 4 by a factor of about 5. This is direct evidence for the behavior of the flat-spectrum source population predicted from Peacock's analyses of the source counts. These show that the maximm comoving density of flat-spectrum sources occurs at redshifts of about 2-2.5 and that the numbers are 
falling off at large redshifts. It is important to emphasize that this sample is selected purely by radio criteria and seems to be producing the same type of distribution found by Dr. Schmidt in the emission-line surveys for radio quiet quasars. 